Bull. Austral. Math. Soc.

VOL. $61(2000)$ [351-352]

\title{
Metrization and Manifolds
}

\section{Abdul Adheem Mowatt Mohammad Al-Soodinay \\ (A.M. MOHAMAD)}

This thesis is devoted to the study of metrizability of generalized metric spaces and manifolds.

The thesis is divided into two parts: the first part presents the theory of generalized metric spaces and metrization theory. The second part is devoted to the topology and algebraic structure of manifolds.

Diagonal properties are studied. We show that they play a central role as factors of metrizability. We use the technique of $g$-maps to develop the theory of generalized metric spaces. The concept of quasi- $G_{\delta}^{*}$-diagonal is introduced and its inter-relationships with generalized metric properties are investigated. We prove that all generalizations of Nagata (respectively $\gamma$ ) spaces considered in this thesis are equivalent to Nagata (respectively $\gamma$ ) spaces if they have a quasi-G*-diagonal. We give a full positive answer for two old questions in developability of Fletcher and Lindgren [1] and an old question of Martin in metrizability [3]. Arhangel'skiî's idea of cleavability is investigated in the context of manifolds in which "perfectness" type properties play a key role. Some mapping theorems are established and questions of Gittings are answered negatively [2].

The theory of non-metrizability of manifolds is studied. It is shown how 'normal' in two well known results, '( $\left.\mathrm{MA}\left(\omega_{1}\right)\right)$ normal, perfect manifolds are metrizable' [6] and [7] and 'normal Moore manifolds are metrizable' [5], can be weakened to 'weakly normal', a very weak separation axiom clearly possessed by spaces cleavable over separable metrizable spaces. We construct two manifolds which answer two of Nyikos's problems [4] in the theory of non-metrizable manifolds. We prove that there is a quasi-developable manifold with a $G_{\delta}$-diagonal, which is not developable; and a consistent example of a quasi-developable, countably metacompact manifold, which has a $G_{\delta}$-diagonal but is not perfect. The two manifolds are highly geometric, unlike most pathological manifolds. In addition, the topology of these two manifolds can be defined "all in one go". We consider the class of $p$-adic analytic manifolds. The wide variety of non-metrizable $p$-adic analytic manifolds is contrasted with the scarcity of metrizable $p$-adic analytic manifolds. The

\section{Received 5th October, 1999}

Thesis submitted to the University of Auckland, April 1999. Degree approved, July 1999. Supervisors: Professor David Gauld and Dr. David McIntyre.

Copyright Clearance Centre, Inc. Serial-fee code: 0004-9727/00 \$A2.00+0.00. 
topology of $p$-adic analytic manifolds is compared with that of real (analytic) manifolds. We present the homeomorphism groups of manifolds, explaining why non-metrizable manifolds are better behaved, with regard to their homeomorphism groups, than metrizable manifolds. A proof that the natural topology on the homeomorphism group for a one dimensional metrizable manifold is the minimum group topology but the homeomorphism group does not admit a minimum group topology for a metrizable manifold of dimensional greater than one is given. We also remark that the usual (compact-open) topology on the homeomorphism group of the Cantor set is the minimum group topology, but that the usual topology on $H\left(I^{\omega}\right)$ is not minimal (this answers a question of Stoyanov [8]). Likewise, examples demonstrating how badly behaved the homeomorphism groups of continua are, in comparison with homeomorphism groups of manifolds, are also given.

\section{REFERENCES}

[1] P. Fletcher and W. Lindgren, 'On $w \Delta$-spaces, $w \sigma$-spaces and $\Sigma^{\sharp}$-spaces', Pacific $J$. Math. 71 (1977), 419-428.

[2] R. Gittings, 'Open mapping theory', in Set-theoretic topology (Academic Press, New York, 1977), pp. 147-191.

[3] H.W. Martin, 'Remarks on the Nagata-Smirnov metrization theorem', in Topology, Proc. Conf. Memphis, Tennessee, 1975 (Marcel Dekker, New York, 1976), pp. 217-224.

[4] P. Nyikos, 'Mary Ellen Rudin's contributions to the theory of nonmetrizable manifolds. The work of Mary Ellen Rudin', Ann. New York Acad. Sci. 705 (1993), 92-113.

[5] M. Reed and P. Zenor, 'Metrization of Moore spaces and generalized manifolds', Fund. Math. 91 (1976), 203-210.

[6] M.E. Rudin, 'The undecidability of the existence of a perfectly normal nonmetrizable manifold', Houston J. Math. 5 (1979), 249-252.

[7] M.E. Rudin and P. Zenor, 'A perfectly normal nonmetrizable manifold'; Houston J. Math. 2 (1976), 129-134.

[8] L. Stoyanov, 'Total minimality of the unitary groups', Math. Z. 187 (1984), 273-283.

Department of Mathematics

The University of Auckland

Private Bag 92019

Auckland

New Zealand

e-mail: mohamad@math.auckland.ac.nz 